\title{
Synchronized Time Profile Similarity in Applications to Nearest Neighbor Classification
}

\author{
Qimin $\operatorname{Liu}^{1}$ \\ Vanderbilt University, Nashville, TN 37240, USA \\ qimin.liu@vanderbilt.edu \\ Acknowledgement: The project was completed when the author was affiliated with \\ the University of Notre Dame.
}

\begin{abstract}
One of the existing approaches to time series classification exploits the time profiles using the original data with synchronization instead of model-implied data. Synchronization aligns inter-individual data from different time points to account for potential phase offsets and nonstationarity in the data. Such synchronization has been applied in psychology: For example, coordinated motion between two individuals exchanging information was used as a predictor and outcome of psychological processes. Synchronization also affords better classification outcomes, as discussed in the data mining community, through aligning the data to reveal the maximally shared profile underlying two compared data sequences. For inter-individual comparison of univariate time series data, existing similarity indices include Euclidean distances and squared correlations. For synchronization, we introduce dynamic time warping and window-crossed lagging. The current study compares the Euclidean distance and the squared correlation before and after synchronization using window-crossed lagging and dynamic time warping in applications to one-nearest-neighbor classification tasks. Discussion, limitations, and future directions are provided.
\end{abstract}

Keywords: classification, time series, dynamic time warping

\section{Introduction}

Advancement in and accessibility to technology have enabled psychologists to harvest big data at greater complexity and larger scale. Intensive longitudinal data are often the product of many repeated measurements through approaches such as daily diary, experience sampling, and burst measurements. The pervasive use of smartphones, fitness trackers, and the Internet of Things have made the use of intensive longitudinal data increasingly accessible. The latitude of information in intensive longitudinal data can enable data-driven investigation of novel research questions. In this paper, we examine classification of timeinvariant membership based on individual trajectories across time. (Harlow \& Oswald, 2016). 
More attention is due on the use of intensive longitudinal data from a big data perspective. Intensive longitudinal data are no stranger to psychological researchers. Daily diary data help developmental psychologists to explore the process of aging (Birditt, Fingerman, \& Almeida, 2005). Clinical psychologists utilize daily data of multiple individuals to explore mental health problems (Laurenceau, Barrett, \& Rovine, 2005). Brain activity measures, such as EEG and fMRI methods, that are frequently employed by cognitive neuroscientists result in data that are collected at high frequency (Kounios \& Beeman, 2009). In addition, organizational and industrial psychologists make use of daily diary data as well to study psychological issues at workplace (Conway \& Briner, 2002). As data of intense repeated measures have already shown great promise in the field of psychology, application of classification and prediction methods may afford researchers and practitioners additional viewpoints and practical utility.

One novel research problem that psychologists may be interested in is time series classification, i.e., to assign time-invariant class membership to test individuals given training time series data of multiple individuals with their respective time-invariant class membership and the time series data of the test individual. For example, cognitive neuroscientists attempted to use the brain activity data to predict the success of word recall tasks (Ezzyat et al., 2017). In particular, Ezzyat and colleagues used time series classification in hope of uncovering the mechanism of episodic memory performance with respect to brain activities and also to help decide whether intracranial brain stimulation should be induced to improve memory performance. As shown in the example, the reason for psychologists to explore the time series classification problem can be two-fold. On the one hand, classification, to some extent, extracts the signals behind the noise from the data and thus can represent the useful information in the data. For example, consider a potential personality theory where personality categorization is based upon time profile. To validate such theory, time series classification can be applied where the classification accuracy may serve to explore the utility and the practicality of such theory. On the other hand, the classification problem can readily translate academic research to real-world applications. For example, clinical psychologists may utilize routine outcome data from behavioral care to aid in diagnosis or in decisions of post-treatment care regime. While approaches for time series classification exist, such as through autoregressive logistic or multinomial models, these conventional methods have limitations.

One limitation in commonly used conventional modeling of intensive longitudinal data, e.g., autoregressive moving average models (Box, Jenkins, \& Reinsel, 2008), lies in its various assumptions. One such assumption is stationarity. Admittedly, stationarity assumption is beneficial both in computation and interpretation: The collected times series information is assumed to be representative of the entire behavior and thus relevant statistics can be meaningfully obtained. In other words, for each person, any interval of time is assumed to be representative for the intra-individual changes. To this end, data preprocessing techniques (West \& Hepworth, 1991) exist to shape the data to better fit the stationarity 
assumption. However, such techniques may mask the important substantive information that the nonstationarity in behavioral time series data may convey. For example, nonstationarity can imply nonconstant dynamics of within-person changes (Boker, Staples, \& Hu, 2016). Moreover, given the big data nature in intensive longitudinal data, the enormous amount of measurement occasions may render the stationarity assumption for the whole series unlikely. Therefore, innovative methods that do not require such assumptions may glean the information that conventional methods often omit due to their assumptions.

While the big data aspect of intensive longitudinal data may be new, psychologists have been interested in class membership in relevance to individual profiles across time. Admittedly, meager efforts have been paid to developing methods for time series classification particular to social and behavioral science: A brief search in selected methodological journals for psychology Structural Equation Model, Psychological Methods, Psychometrika, Multivariate Behavioral Research, Behavioral Research Methods with keyword time series classification returned no exact match. However, quantitative psychologists have made relevant discussions. For example, Gates et al. (2017) discussed community detection within group iterative multiple model estimation for clustering time series data. The goal was to simultaneously detect homogeneous subgroups and to classify individuals to identified groups. This is often referred to as a clustering problem that is "unsupervised" as it does not require a training data with assigned class membership information (Gates, Lane, Varangis, Giovanello, \& Guiskewicz, 2017). The focus of this paper, however, is on classification problems that are supervised: The training data contain both time series information and the timeinvariant class membership labels; the goal is to predict the membership for new or test data given time series information. In other words, provided with time profile and their subgroup labels, we aim to "learn" the difference between predefined subgroups and apply this to classify individuals into predefined subgroups.

Inter-individual differences in intra-individual time profiles are central to discerning between predefined classes. For classification algorithms to exploit the inter-individual differences of the training data and to discover the pattern to apply to the test data, inter-individual similarity measures of time series data can be highly relevant. The intricacy in time series data asks methodological researchers not only to consider simple similarity indices but also to take into account of the potential nonsynchrony between time profiles. That is, two time profiles can look different just because the processes are not in sync or the individuals in comparison are not aligned at their respective phases. For example, in psychotherapy process research, individuals may be studying different cognitive behavioral skills modules. The daily diary data between individuals may show different patterns despite the fact that they would be the same if the individuals were undergoing the same module.

The alignment and synchrony of time series data between individuals can convey substantive meaning to psychologists. For example, interpersonal communication can often come with certain levels of coordinated behaviors, such as 
nonverbal synchrony. Nonverbal synchrony can be that where eye gaze or facial expressions are in sync between individuals. For example, lack of eye contact in communications can be seen as lack of nonverbal synchrony and can be interpreted as insincere (Ramseyer \& Tschacher, 2006). Nonverbal synchrony has been employed to investigate association between nonverbal behaviors and psychotherapeutic outcomes (Ramseyer \& Tschacher, 2011). Although the idea of nonsynchrony seemed to have only applied to study dyadic time series data, the idea should transcend into studying the inter-individual similarity of time profiles in general. Furthermore, it is worth noting that quantitative psychologists have proposed window cross-correlation (WCC) to measure such nonsynchrony, which breaks the data into segments of window and computes a correlation matrix across windows (Boker, Xu, Rotondo, \& King, 2002). In fact, phase misalignment or nonsynchrony have also been entertained in the machine learning community (Jeong, Jeong, \& Omitaomu, 2011).

Machine learning community has offered a great amount of discussion in time series classification in general with over 100 methods before 2003 and even more dedicated efforts in recent years (Bagnall, Lines, Bostrom, Large, \& Keogh, 2017). One benchmark method, dynamic time warping (DTW) one nearest neighbor $(1 \mathrm{NN})$, pays special attention to phase alignment before computing similarities between individuals (Sakoe \& Chiba, 1978). In short, dynamic time warping involves maximally aligning the two times series prior to comparison. Moreover, dynamic time warping, combined with nearest neighbor classification, has shown success for real-world applications such as in human physical activity detection (Sempena, Maulidevi, \& Aryan, 2011). However, the performance of DTW $1 \mathrm{NN}$ in social and behavioral time series classification tasks, to the author's knowledge, has not been investigated.

The present paper aims to continue exploring the big data aspect of intensive longitudinal data in social and behavioral sciences. In particular, we provide discussions on supervised time series classification. Since the stationarity assumption may not hold for intensive longitudinal data, we limit most of our discussions to methods that do not assume stationarity. Moreover, given the potential substantive meaning of phase nonsynchrony and misalignment in social and behavioral sciences, we only discuss techniques that include such consideration. The rest of the paper is organized as follows. First, we review two general inter-individual similarity measures, Euclidian distance and squared correlation, as the former is used in DTW and the latter in WCC. Then, we introduce two techniques for phase alignment, DTW and window crossed lagging (which is derived from WCC). After that, we provide details on pairing phase alignment methods with similarity measures in applications to $1 \mathrm{NN}$ for time series classification. Furthermore, we provide a simulation study to show the potential of discussed methods. The paper aims to motivate psychologists to consider time series classification problems. In addition, since dynamic time warping has mostly been applied only to Euclidean distances and window cross lagging only to correlations, the present paper also innovates by disaggregating phase alignment methods to similarity indices and introduces some example indices via 
mixing-and-matching existing techniques. Lastly, we hope to promote the use of dynamic time warping to psychologists and window cross lagging to the machine learning community.

\section{Methods}

\subsection{Similarity Measures}

Euclidean distance, $d$, between two points $x_{i}$ and $x_{j}$ is defined as the length of the shortest straight-line segment that connects the two points, $d=\left|x_{i}-x_{j}\right|$. The Euclidean distance between two vectors $X_{i}=\left(x_{i}^{(1)}, x_{i}^{(2)}, \ldots, x_{i}^{(T)}\right)$ and $X_{j}=$ $\left(x_{j}^{(1)}, x_{j}^{(2)}, \ldots, x_{j}^{(T)}\right)$ is subsequently defined as $d\left(X_{i}, X_{j}\right)=$

$$
\sqrt{\left(x_{i}^{(1)}-x_{j}^{(1)}\right)^{2}+\left(x_{i}^{(2)}-x_{j}^{(2)}\right)^{2}+\ldots+\left(x_{i}^{(T)}-x_{j}^{(T)}\right)^{2}}=\sqrt{\sum_{t=1}^{T}\left(x_{i}^{t}-x_{j}^{t}\right)} .
$$

Here, $i$ and $j$ denote individuals $i$ and $j, T$ is the total time points, and $t$ represents specific time points. It is worth noting that, first, to calculate Euclidean distance between two vectors, two vectors should share the same length; second, the Euclidean distance of two vectors sums the distance between concurrent points before the square root. Euclidean distance can be used to measure data points of two individuals or data sequences of two individuals. The magnitude of Euclidean distance is inversely related with the similarity between two individuals.

Squared correlation, $r^{2}$, between two vectors $X_{i}=\left(x_{i}^{(1)}, x_{i}^{(2)}, \ldots, x_{i}^{(T)}\right)$ and $X_{j}=\left(x_{j}^{(1)}, x_{j}^{(2)}, \ldots, x_{j}^{(T)}\right)$ can be given by $r^{2}\left(X_{i}, X_{j}\right)=\left(\frac{\sum\left(x_{i}^{(t)}-\bar{x}_{i}\right)\left(x_{j}^{(t)}-\bar{x}_{j}\right)}{T \times s_{x_{i}} s_{x_{j}}}\right)^{2}$. The squared correlation of data sequences between individuals can represent the similarity. Similar to Euclidean distance, the above formula requires two data sequences to be of equal length. While Euclidean distance varies with the absolute value of the data, squared correlation is invariant to linear transformation.

\subsection{Phase Alignment: Dynamic Time Warping}

The goal is to compare two time-dependent sequences $X_{i}=\left(x_{i}^{(1)}, x_{i}^{(2)}, \ldots, x_{i}^{(T)}\right)$ of length $T_{i} \in \mathbb{N}$ and $X_{j}=\left(x_{j}^{(1)}, x_{j}^{(2)}, \ldots, x_{j}^{(T)}\right)$ of length $T_{j} \in \mathbb{N}$. Graphically, for two sequences to appear similar in shape and thus phase-aligned, each pair of concurrent points should only show small differences in Euclidean distance, which would in turn make two sequences close to each other visually. Euclidean distance helps measure the visual proximity between two points because the Euclidean distance is the shortest straight-line distance. Dynamic time warping first originates from speech recognition literature (Sakoe \& Chiba, 1978). In 
speech recognition, the speech data of the same words may appear different for different utterance, for example, the pace of how one talks or the length of pause between words. Thus, the speech data sequence of two individuals may appear out of phase due to the difference in pause and the length of the phase for two individuals may also differ owing to the difference in talking speed. Dynamic time warping attempts to adjust for such phase misalignment by stretching or shrinking each phase so that two data sequences are maximally in sync in a sense that two data sequences would appear similar graphically.

Dynamic time warping thus attempts to align each point in one sequence to each closest points in the other sequence under some constraints. Let $d(m, n):=$ $d\left(x_{i}^{m}, x_{j}^{n}\right)$ denote the Euclidean distance between a pair of elements in $X_{i}$ and $X_{j}$. A $\left(T_{i}, T_{j}\right)$-warping path between $X_{i}$ and $X_{j}, p=\left(p_{1}, \ldots, p_{L}\right)$ where $p_{l}=$ $\left(m_{l}, n_{l}\right) \in\left[1: T_{i}\right] \times\left[1: T_{j}\right]$ for $l \in[1, L]$. That is, each element in the warping path, $p$, record the pairing of an element in $X_{i}$ to in $X_{j}$. The warping path satisfies the following conditions:

1. Boundary condition: $p_{1}=(1,1)$ and $p_{l}=\left(T_{i}, T_{j}\right)$

2. Monotonicity condition: $n_{1} \leq n_{2} \leq \ldots \leq n_{L}$ and $m_{1} \leq m_{2} \leq \ldots \leq m_{L}$, i.e., $n_{t} \leq n_{t^{\prime}}$ and $m_{t} \leq m_{t^{\prime}} \forall t<t^{\prime}$ where $t, t^{\prime} \in[1, L] \cap \mathbb{N}$

3. Step size condition: $p_{l+1}-p_{l} \in\{(1,0),(0,1),(1,1)\}$ for $l \in[1, L-1] \cap \mathbb{N}$

The first condition requires the first and the last element in $X_{i}$ and in $X_{j}$ are always respectively matched to each other so that all elements of two sequences are aligned. The second condition helps the alignment proceed forward. The third condition further specifies the alignment process and requires that no elements can be omitted and that no path step can involve the same pair.

After alignment, $X_{i}$ and $X_{j}$ are augmented into $X_{i}^{*}=\left(x_{i}^{1}, x_{i}^{m_{l}}, \ldots, x_{i}^{T_{i}}\right)$ and $X_{j}^{*}=\left(x_{j}^{1}, x_{j}^{n_{l}}, \ldots, x_{j}^{T_{j}}\right)$. The distance between $X_{i}^{*}$ and $X_{j}^{*}$ can be defined as $\Delta\left(X_{i}^{*}, X_{j}^{*}\right)=\sum_{l=1}^{L} d\left(m_{l}, n_{l}\right)$. An optimal warping path is that which $\Delta\left(X_{i}^{*}, X_{j}^{*}\right)$ is at its minimum, that is, $\operatorname{DTW}\left(X_{i}^{*}, X_{j}^{*}\right)=\operatorname{ArgMin} \Delta\left(X_{i}^{*}, X_{j}^{*}\right)$ given that $p$ satisfies the conditions of a warping path. Let $\Delta^{*}(m, n)$ denote the optimized DTW distance between $X_{i}^{*}$ and $X_{j}^{*}$ up to point the $m$ th and the $n$th element respectively, and the optimization can be realized in the following algorithm:

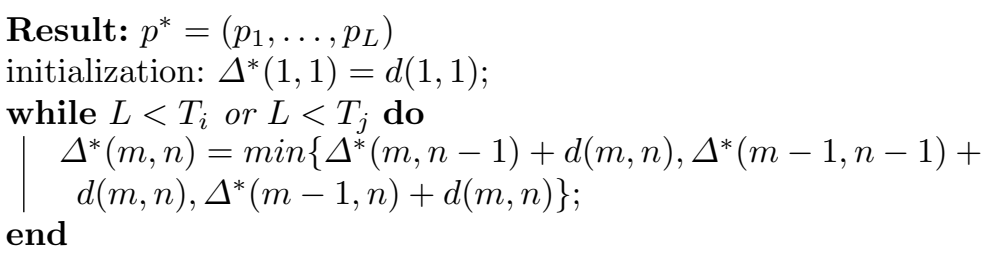

The algorithm effectively yields the optimal path $p^{*}=\left(p_{1}, \ldots, p_{L}\right)$ from the reverse order of the indices starting with $p_{L}=\left(T_{1}, T_{2}\right)$. Let the previous step $l$ be known, $p_{l}=\left(m_{l}, n_{l}\right)$. Note that the optimal path is defined backwards: The initial step is $p_{L}$, which is the alignment of last elements in both sequences. 
The optimal step next step $l-1$ is $p_{l-1}=\operatorname{Argmin}\left\{d\left(m_{l}-1, n_{l}-1\right), d\left(m_{l}-\right.\right.$ $\left.\left.1, n_{l}\right), d\left(m_{l}, n_{l}-1\right)\right\}$. In the case where $m_{l}=1, p_{l-1}=\left(1, n_{l}-1\right)$. Similarly, $p_{l-1}=\left(m_{l}-1,1\right)$ if $n_{l}=1$. The optimal path is defined when $l$ reaches 1 , that is, $p_{1}=(1,1)$. It is worth noting that the minimum value may not be unique. That is, multiple optimal warping paths may exist. However, while the specific paths differ, the resulting distance $D T W\left(X_{i}^{*}, X_{j}^{*}\right)$ would be the same for all optimal warping paths.

We illustrate the algorithm with a numerical example. Consider

$$
\begin{aligned}
& X_{1}=(1, .77, .17,-.5,-.94,-.94,-.5, .17, .76,1) \\
& X_{2}=(.33, .68,1.04, .96, .36,-.25,-.77,-.92,-.58,0)
\end{aligned}
$$

The raw sequences are plotted in 2.2. To find an optimal warping path $p^{*}$, we start with $p_{L}=(10,10)=d(10,10)=1$. Thus, the next step is,

$$
\begin{aligned}
p_{L-1} & =\operatorname{Argmin}\{d(10,9), d(9,10), d(9,9)\} \\
& =\operatorname{Argmin}\{|1-(-.58)|,|.76-0|,|.76-(-.58)|\} \\
& =\operatorname{Argmin}\{1.58, .76,1.34\} \\
& =(9,10) .
\end{aligned}
$$

A similar procedure can be repeated till the optimal warping path $p^{*}$ is fully defined. One potential optimal path is $p^{*}=\{(10,10),(10,9),(10,8),(9,7),(8,6)$, $(8,5),(7,5),(6,4),(5,3),(4,2),(3,1),(2,1),(1,1)\}$. The DTW aligned sequences are also plotted in 2.2. As shown in 2.2, the DTW results in phase alignment that reveals a maximally shared and synced graphical pattern. Subsequently, to compare two sequences, the Euclidean distance, for example, can be calculated between DTW aligned $X_{1}^{*}$ and $X_{2}^{*}$ given $p^{*}$. DTW can be readily implemented in $\mathrm{R}$ using the package dtw (Giorgino, 2009). Given sequences $\mathrm{x}$ and $\mathrm{y}, \mathrm{dtw}(\mathrm{x}, \mathrm{y})$ computes euclidean distance after dynamic time warping. To extract the distance, we can use dtw $(x, y) \$$ normalizedDistance. Let align $=d_{t w}(x, y)$, we can use cor (x [align\$index1], y[align\$index 2$])^{\wedge} 2$ to compute squared correlation with dynamic time wapring alignment.

\subsection{Phase Alignment: Window Cross Lagging}

Window cross lagging (Boker et al., 2002) assumes stationarity in short durations of the time series. By breaking a time-dependent data sequences into smaller windows, stationarity assumption is more likely to be met than for the entire sequence. Thus, this may prove particularly beneficial for intensive longitudinal data. Given stationarity, any collection of measurement occasions within a window is representative of the window. Moreover, all occasions of measurement within a window share an underlying expected value that does not vary across occasions within the window. As such, common statistics such as means and variances can be meaningfully computed. In particular, a window, $W_{x_{i}}$, is defined as a sequential measurements sampled from the time series $X_{i}$. For a 

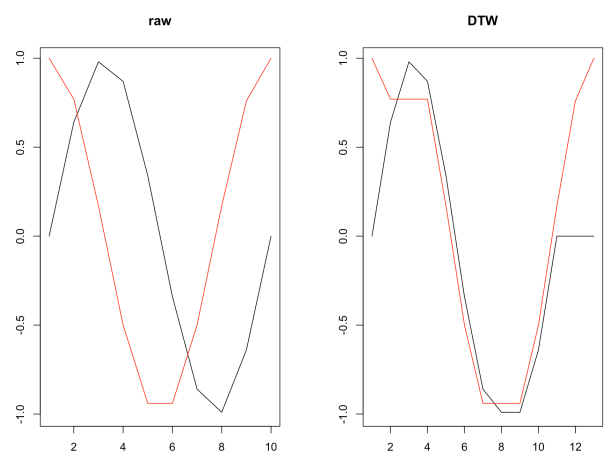

Fig. 1. Illustrative Example of DTW.The left plot shows data sequences before alignment and the right plot shows data sequences after alignment. The red line represents $X_{1}$ and the black line represents $X_{2}$. The $\mathrm{X}$-axis is the index of the number. Note that DTW "stretches" the sequence, resulting in greater maximum index. The Y-axis represents the value of the datum.

data sequence of length $T$ and windows of size $T_{W}$, we can identify $T-T_{W}+1$ windows.

The window size can be theoretically determined. For example, a 13-week window, the median mood cycle (Solomon et al., 2010), can be chosen so that the windowing is informative given its substantive underpinning. This can be both advantageous and disadvantageous: The theoretical basis can make the interpretation of statistics from windowing more meaningful; however, the strength of the theory may also relate to the quality of similarity metrics from windowing. Windowing let researchers specify the length of the phase given theoretical reasons. It is worth noting the specified phases are of the same length and at times can be at odds with theoretical considerations.

After obtaining the windows from data sequences in comparison, instead of one single comparison to be made in other regular comparison like in Euclidean distance, a matrix of similarity metrics can be obtained through crosscomparison. Given a desired lag range of size $v$, the ath window in the first sequence can be compared to $(a-v)$ th, $(a-v+1)$ th, ... $(a+v)$ th window of the other sequence. Each window can be compared to $2 v+1$ windows in the other sequence. Specifically, for example, a $v=1$ cross-lagged comparison would be comprised of three comparisons: the comparison between the ath window of the first sequence to the $a-1$ th window of the second sequence, the comparison between the $a$ th windows of both sequences, and the comparison between the $a-1$ th window of the first sequence and the $a$ th window of the second sequence. The original paper used correlation as the metric (Boker et al., 2002) where each element of the matrix is the correlation of respectively lagged windows. The correlation between two windows can be expressed as $r\left(W_{x_{i}}, W_{x_{j}}\right)=\frac{1}{T_{w}} \sum_{t=1}^{T_{W}} \frac{\left(W_{x_{i}}^{(t)}-\bar{W}_{x_{i}}\right)\left(W_{x_{j}}^{(t)}-\bar{W}_{x_{j}}\right)}{s_{W_{x_{i}}} s_{W_{x_{j}}}}$ 
We illustrate the window cross lagging procedure in terms of the window cross correlation. Recall prior example of two data sequences $X_{1}$ and $X_{2}$. We consider a lag range of size 1 and window size of 8 for the sake of convenience. First, the windowing step resulted in 3 windows for both data sequences. The specific windows are displayed in Table 1 Left. Then, the cross lagging procedure resulted in a $3 \times 2$ matrix as shown in Table 1 Right.

\begin{tabular}{ccccccc}
\multicolumn{1}{c}{$\mathrm{X}_{1}$} & \multicolumn{3}{c}{$\mathrm{X}_{2}$} & & \multicolumn{2}{c}{ lag } \\
\hline Window 1 & $1, .77, .17,-.5,-.94,-.94,-.5, .17$ & $.33, .68,1.04, .96, .36,-.25,-.77,-.92$ & & -1 & 0 & 1 \\
Window 2 & $.77, .17,-.5,-.94,-.94,-.5, .17, .76$ & $.68,1.04, .96, .36,-.25,-.77,-.92,-.58$ & & -.59 & .00 & .69 \\
Window 3 & $.17,-.5,-.94,-.94,-.5, .17, .76,1$ & $1.04, .96, .36,-.25,-.77,-.92,-.58,0$ & & -.69 & -.14 & .59
\end{tabular}

Table 1. Left: Example Windowing with $T_{w}=8$; Right:WCC with $v=1$ and $T_{w}=8$

Since window cross lagging results in a matrix of similarity metrics, summarizing the matrix into a single index may be preferable especially in applications to nearest neighbor classifier. A number of strategies can be considered. One strategy is to compute the average of all matrix elements. Such averages can represent the average similarity between two sequences across phase alignments. Another is to pick meaningful elements from the matrix. For example, with WCC, one may desire to choose the maximum squared correlation across all elements. The maximum squared correlation may represent the strongest signal across lags and between phases. Similarly, if Euclidean distance is coupled with window cross lagging, then the minimum may be chosen as small Euclidean distance indicate high similarity. The following $\mathrm{R}$ scripts implement aforementioned indices:

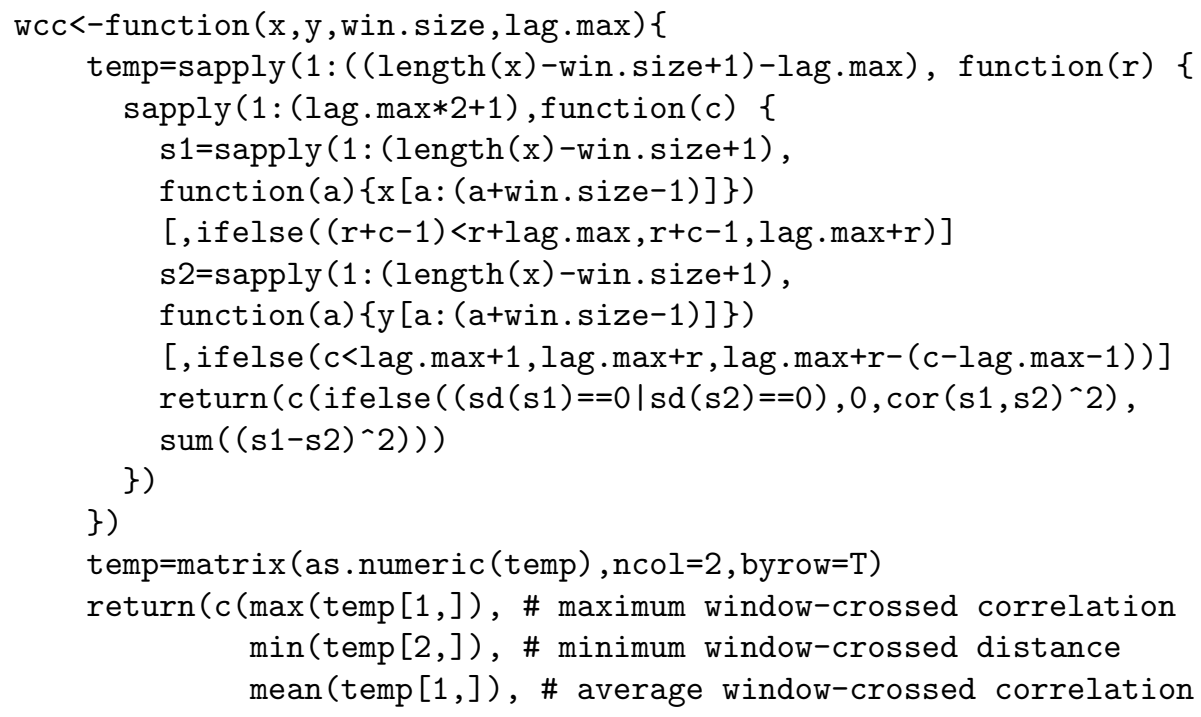




\subsection{Nearest Neighbor Classification}

Given a similarity measure of two time-dependent sequences that handles phase misalignment, nearest neighbor classification can be readily used. Consider a training dataset of size $n, S=\left\{X_{1}, X_{2}, \ldots, X_{n}\right\}$ where any element in $S$ is a time-dependent sequence measured across $T$ occasions, $X_{i}=\left(x_{i}^{(1)}, x_{i}^{(2)}, \ldots, x_{i}^{(T)}\right)$. Moreover, for each $X_{i}$, we are also given the class membership information $Y_{i}$. Now we wish to determine the class membership of a test data sequence $X_{j}$. The logic behind nearest neighbor (NN) classification is that observations of the same class ought to be similar to each other. In the context of time series classification, we expect time-dependent sequences of the same class to show similar patterns after adjusting for phase misalignment. The algorithm of NN classification can be summarized as follows,

1. Compute the distance $\delta$ between $X_{j}$ and each element in $S$, i.e., $X_{i}$ for all is in $[1, n] \cap \mathbb{N}$. That is, we obtain $D_{j}=\left\{\delta\left(X_{j}, X_{1}\right), \delta\left(X_{j}, X_{2}\right), \delta\left(X_{j}, X_{n}\right)\right\}$ where $\delta$ can be any distance measure such as Euclidean distance, squared correlation, DTW distance, DTW-aligned squared correlation, mean WCC, or mean window cross-lagged distance (WCD).

2. Rank the vector of distance, $D_{j}$, in order to identify the nearest neighbor(s). Denote the ranked distance vector as $D_{j}^{*}$. Based on the choice of $\delta$, the $k$ nearest neighbor(s) are those with $k$ largest or smallest $\delta$. We denote $N$ as the set of indices of these nearest neighbors. For example, because small Euclidean distance indicates high similarity, the $k$ nearest neighbor would be the $k X_{i}$ s with smallest $\delta$.

3. Assign the most frequently counted class membership among the identified nearest neighbors as the predicted class membership for $X_{j}$. That is, let $F$ denote the frequencies for each class within $\left\{Y_{i}\right\}$ with $i \in \mathbb{N}, \hat{Y}_{j}=\operatorname{argmax} F$.

In the context of time series classification, $1 \mathrm{NN}$ is especially popular. $1 \mathrm{NN}$ is a nearest neighbor classifier where only the single nearest neighbor is identified and the class membership of the new observation is assigned as the class membership of the single nearest neighbor. Such popularity is not unjustified. On the one hand, the computation of the similarity index between time-dependent sequences is costly. For example, the computational cost of DTW is $O\left(T_{1} T_{2}\right)$ and of WCC is $O\left((2 v+1)\left(T-T_{w}+1\right)\right)$. Using $\mathrm{kNN}$ with $k$ from cross-validation would further the computational cost and render the classification task resource-, time, computation-consuming. On the other hand, it has been shown that crossvalidated $\mathrm{kNN}$, at least with DTW distances and with Euclidean distances, did not show significant improvement over 1NN (Bagnall \& Lines, 2014). Admittedly, it hasnt been tested if cross-validated $\mathrm{kNN}$ with window cross-lagged metrics 
would perform significantly better than $1 \mathrm{NN}$. For comparability, we only consider $1 \mathrm{NN}$ in the present paper for all distance metrics.

To implement $1 \mathrm{NN}$ in R, let dist be a distance matrix where each column represents a case from the training dataset and each row represents a case from the test dataset. Let $y$ be the labels from the training dataset. dist $[i, j]$ is the distance between $i$ th observation in the test dataset and $j$ th observation in the training dataset. To determine the label for the $i$ th observation in the test dataset, we use y[which.min(dist [i])] as the predicted label.

\section{Simulation Design and Data Generation}

Our first simulation study was designed to show the performance of $1 \mathrm{NN}$ with mix-and-match similarity measures with and without phase alignment with ARbased classes. In particular, we considered two similarity measures without phase alignment: squared correlation (sqrC) and Euclidean distance (EucD). We considered two similarity measures with DTW: DTW distance (DTWd) where Euclidean distance was calculated after DTW alignment and DTW squared correlation (DTWc). We also considered four similarity measures with window cross lagging: the average of all elements in the WCC matrix (WCCmean), the maximum of the WCC matrix (WCCmax), the average of all elements in window cross-lagged Euclidean distance matrix (WCDmean), and the minimum of the window cross-lagged Euclidean distance matrix (WCDmin).

In addition, we included a simple method based on an autoregressive (AR) model: we first fitted an $\operatorname{AR}(1)$ model to all $X_{i}$ in the training dataset $S$ and obtained the AR coefficient $\rho_{i}$ S and then computed the empirical density function (edf) in each class. After that, we applied the $\operatorname{AR}(1)$ coefficient $\rho_{j}$ of the test case $X_{j}$ to the edfs for each class, obtained the probability of $\rho_{j}$ being in the distribution of $\operatorname{AR}(1)$, and subsequently assigned the class membership for $X_{j}$ as from that where the edf had the highest probability from fitting $\rho_{j}$. The ARbased method differs from $1 \mathrm{NN}$ with aforementioned similarity measures in that AR-based methods assume a parametric form and stationarity for the whole time series.

In particular, three classes were present in our simulation study. (1) Random noise class: The time series data were simply random noises without any time-dependent pattern; (2) $\mathrm{AR}(1)$ class: The times series data in this class were generated with realistic model parameters from an AR(1) model (Wang, Hamaker, \& Bergeman, 2012) (3) The nonstationary class where the data still showed a time-dependent pattern but do not meet the stationarity assumption.

The data were generated using the model $x_{i}^{(t+1)}=x_{i}^{(t)} \rho_{i} Y_{i}+\varepsilon^{(t+1)}$ where the AR coefficient $\rho_{i} \sim N(.2, .2)$ and the error term $\varepsilon^{(t+1)} \sim N(0,1)$. Moreover, $Y_{i} \in 0,1,5$, respectively corresponding to the random noise, $\operatorname{AR}(1)$ process, and a nonstationary class. 
One variable was manipulated: The balance between training samples per class. For the balanced condition, training datasets with a sample size of 150 were generated with each class of 50 observations. For the imbalanced condition, the random noise, $\operatorname{AR}(1)$, and the nonstationary class respectively had 30, 80, and 40 observations. For both conditions, a test dataset of 30 observations with 10 observations for each class was used to evaluate the performance of different classification algorithms. 500 replications were conducted.

Performance was evaluated via the average overall accuracy and the average accuracy per class, which is computed via the mean of percentages of the true positives and the true negatives per class.

\section{Results and Discussion}

The results for the balanced condition and the imbalanced condition are summarized respectively in Table 2 . The balance of training samples per class had some effect on the overall accuracy with the imbalanced condition having higher overall accuracy in general. For example, overall accuracy for all methods were above $70 \%$ for the imbalanced condition while only WCDmin exceeded $70 \%$ accuracy for the balanced condition. This observation encourages future study to examine the effect of balance in greater detail where different setup of imbalance should be investigated.

\begin{tabular}{ccccccccc} 
& \multicolumn{4}{c}{ Balanced } & \multicolumn{5}{c}{ Imbalanced } \\
& Overall Random noise & AR(1) & Nonstationary & Overall Random noise AR(1) Nonstationary \\
\hline EucD & 0.61 & 0.48 & 0.92 & 0.43 & 0.71 & 0.67 & 0.78 & 0.67 \\
DTWd & 0.45 & 0.33 & 1 & 0.01 & 0.77 & 0.67 & 0.98 & 0.67 \\
WCDmin & 0.76 & 0.67 & 0.77 & 0.82 & 0.7 & 0.67 & 0.76 & 0.67 \\
WCDmean & 0.68 & 0.67 & 0.77 & 0.6 & 0.7 & 0.66 & 0.78 & 0.67 \\
sqrC & 0.55 & 0.44 & 0.92 & 0.3 & 0.72 & 0.66 & 0.83 & 0.66 \\
DTWc & 0.54 & 0.45 & 0.84 & 0.33 & 0.71 & 0.67 & 0.78 & 0.67 \\
WCCmax & 0.68 & 0.66 & 0.76 & 0.61 & 0.71 & 0.67 & 0.78 & 0.67 \\
WCCmean & 0.68 & 0.66 & 0.76 & 0.61 & 0.71 & 0.67 & 0.78 & 0.67 \\
AR & 0.65 & 0.66 & 0.81 & 0.49 & 0.78 & 0.67 & 1 & 0.67
\end{tabular}

Table 2. Simulation Results. The table includes results from both the balanced and the imbalanced design. The overall column provides the classification accuracy across all classes. Then per-class accuracy is provided in subsequent columns.

For the balanced condition, WCDmin showed the highest overall performance ( $76 \%$ accuracy) and DTWc performed the worst (54\% accuracy). The result is encouraging because WCD with $1 \mathrm{NN}$ seems to show promise above and beyond the DTW-based measures such as DTWd, which is held as the state-ofthe-art method in the machine learning community. Admittedly, only one datagenerative process was examined in the simulation study. Nevertheless, the result from the simulation study invites researchers to test the performance of similarity measures with window cross lagging in broader contexts. With respect to 
particular classes, WCDmin and WCDmean performed best in identifying the random noise class (67\%), DTWd for the AR(1) class (100\%) and WCDmin for the nonstationary class. It appears that DTWd performed worst for both the random noise class (45\%) and for the nonstationary class (1\%). The performance of DTWd is surprising and future research should study the performance of DTW-based similarity measures specific to nonstationary time profile patterns. Moreover, it is worth discussing that the AR-based method showed only mediocre performance overall $(65 \%)$ even with respect to identifying the $\mathrm{AR}(1)$ class $(81 \%)$.

For the imbalance condition, the AR-based method showed superior performance $(78 \%)$ overall and in identifying the $\operatorname{AR}(1)$ class $(100 \%)$. The second best method with regard to overall accuracy is DTWd (77\%) owing to its good performance in identifying the $\mathrm{AR}(1)$ class (98\%). All other methods showed similar performance both in overall accuracy (around 70\%) and in accuracy per class. The results from the imbalance class should be generalized with caution as only one imbalance setup was considered and the setup may have shown favor towards the $\mathrm{AR}(1)$ class. Because more training samples are allocated to the $\mathrm{AR}(1)$ class, methods that are good at identifying AR(1) class can reach saturated performance. Similarly, with fewer training samples in other classes, it is possible not enough training samples exist to discriminate performance between methods.

\section{References}

Bagnall, A., \& Lines, J. (2014, 6). An Experimental Evaluation of Nearest Neighbour Time Series Classification (Tech. Rep.). Retrieved from http://arxiv.org/abs/1406.4757

Bagnall, A., Lines, J., Bostrom, A., Large, J., \& Keogh, E. (2017, 5). The great time series classification bake off: a review and experimental evaluation of recent algorithmic advances. Data Mining and Knowledge Discovery, 31(3), 606-660. Retrieved from http://link.springer.com/10.1007/ s10618-016-0483-9 doi: 10.1007/s10618-016-0483-9

Birditt, K. S., Fingerman, K. L., \& Almeida, D. M. (2005). Age Differences in Exposure and Reactions to Interpersonal Tensions: A Daily Diary Study. Psychology and Aging, 20(2), 330-340. Retrieved from http://doi.apa.org/ getdoi. cfm?doi=10.1037/0882-7974.20.2.330 doi: 10.1037/0882-7974 .20 .2 .330

Boker, S. M., Staples, A. D., \& Hu, Y. (2016). Dynamics of Change and Change in Dynamics. Journal for person-oriented research, 2(1-2), 34-55. Retrieved from https://www.ncbi.nlm.nih.gov/pubmed/ 29046764https://www.ncbi.nlm.nih.gov/pmc/articles/PMC5642952/ doi: $10.17505 /$ jpor.2016.05

Boker, S. M., Xu, M., Rotondo, J. L., \& King, K. (2002). Windowed crosscorrelation and peak picking for the analysis of variability in the association 
between behavioral time series. Psychological Methods, 7(3), 338-355. doi: 10.1037//1082-989X.7.3.338

Box, G. E. P., Jenkins, G. M., \& Reinsel, G. C. (2008). Time Series Analysis: Forecasting and Control. Wiley. Retrieved from https://books.google . com/books?id=1JnnPQAACAAJ

Conway, N., \& Briner, R. B. (2002). A daily diary study of affective responses to psychological contract breach and exceeded promises (Vol. 23) (No. 3). doi: $10.1002 /$ job.139

Ezzyat, Y., Kragel, J. E., Burke, J. F., Levy, D. F., Lyalenko, A., Wanda, P., .. Kahana, M. J. (2017, 5). Direct Brain Stimulation Modulates Encoding States and Memory Performance in Humans. Current Biology, 27(9), 1251-1258. Retrieved from http://linkinghub.elsevier.com/ retrieve/pii/S0960982217303263 doi: 10.1016/j.cub.2017.03.028

Gates, K. M., Lane, S. T., Varangis, E., Giovanello, K., \& Guiskewicz, K. (2017). Unsupervised Classification During Time-Series Model Building. Multivariate Behavioral Research, 52(2), 129-148. Retrieved from https:// doi.org/10.1080/00273171.2016.1256187 doi: 10.1080/00273171.2016 .1256187

Giorgino, T. (2009). Computing and Visualizing Dynamic Time Warping Alignments in R: The dtw Package. Journal of Statistical Software, 31(7), 124. Retrieved from http://www.jstatsoft.org/v31/i07\%5Cnhttp:// www.jstatsoft.org/v31/i07/ doi: 10.18637/jss.v031.i07

Harlow, L. L., \& Oswald, F. L. (2016). Big data in psychology: Introduction to the special issue. Psychological Methods, 21(4), 447-457. Retrieved from http://doi.apa.org/getdoi.cfm?doi=10.1037/met0000120 doi: $10.1037 /$ met0000120

Jeong, Y.-S., Jeong, M. K., \& Omitaomu, O. A. (2011). Weighted dynamic time warping for time series classification. Pattern Recognition, 44, 2231-2240. doi: $10.1016 /$ j.patcog.2010.09.022

Kounios, J., \& Beeman, M. (2009, 8). The Aha! Moment. Current Directions in Psychological Science, 18(4), 210-216. Retrieved from http://journals .sagepub.com/doi/10.1111/j.1467-8721.2009.01638.x doi: 10.1111/ j.1467-8721.2009.01638.x

Laurenceau, J. P., Barrett, L. F., \& Rovine, M. J. (2005). The interpersonal process model of intimacy in marriage: A daily-diary and multilevel modeling approach. Journal of Family Psychology, 19(2), 314-323. doi: 10.1037/0893-3200.19.2.314

Ramseyer, F., \& Tschacher, W. (2006). Synchrony: A core concept for a constructivist approach to psychotherapy. Constructivism in the human sciences, 11(1-2), 150-171. Retrieved from http://www.researchgate.net/publication/215507443_Synchrony_A _Core_Concept_for_a_Constructivist_Approach_to_Psychotherapy/ file/3606d2cadc7a8d399c757cbb48c1e8ec.pdf doi: 10.1007/ 978-3-642-12397-9-15

Ramseyer, F., \& Tschacher, W. (2011). Nonverbal synchrony in psychotherapy: 
Coordinated body movement reflects relationship quality and outcome. Journal of Consulting and Clinical Psychology, 79(3), 284-295. doi: 10 $.1037 / \mathrm{a} 0023419$

Sakoe, H., \& Chiba, S. (1978). Dynamic Programming Algorithm Optimization for Spoken Word Recognition. IEEE Transactions on Acoustics, Speech, and Signal Processing, 26 (1), 43-49. doi: 10.1109/TASSP.1978.1163055

Sempena, S., Maulidevi, N. U., \& Aryan, P. R. (2011). Human action recognition using Dynamic Time Warping. Electrical Engineering and Informatics (ICEEI), 2011 International Conference on(July), 1-5. doi: 10.1109/ICEEI.2011.6021605

Solomon, D. A., Leon, A. C., Coryell, W. H., Endicott, J., Li, C., Fiedorowicz, J. G., ... Keller, M. B. (2010, 4). Longitudinal Course of Bipolar I Disorder. Archives of General Psychiatry, 67(4), 339. Retrieved from http://archpsyc. jamanetwork. com/article.aspx?doi=10.1001/ archgenpsychiatry.2010.15 doi: 10.1001/archgenpsychiatry.2010.15

Wang, L. P., Hamaker, E., \& Bergeman, C. S. (2012). Investigating interindividual differences in short-term intra-individual variability. Psychological Methods, 17(4), 567-581. Retrieved from http://doi.apa.org/ getdoi.cfm?doi=10.1037/a0029317 doi: 10.1037/a0029317

West, S. G., \& Hepworth, J. T. (1991). Statistical issues in the study of temporal data: daily experiences. Journal of personality, 59(3), 609-662. doi: 10 $.1111 / \mathrm{j} .1467-6494.1991 . t b 00261 . x$ 